\title{
Avaliação in vitro da resistência de união de diferentes combinações entre adesivos e resinas compostas
}

\section{In vitro evaluation of the bond strength of different combinations of adhesives and composite resins}

\author{
Eduardo Batista FRANCO* \\ Maria Silvia de Lima PAZIM** \\ Carlos Eduardo FRANCISCHONE*
}

\begin{abstract}
FRANCO, E. B.; PAZIM, M. S. L.; FRANCISCHONE, C. E. Avaliação in vitro da resistência de união de diferentes combinações entre adesivos e resinas compostas. Pesqui Odontol Bras, v. 14, n. 3, p. 225-231, jul./set. 2000.

O objetivo desta pesquisa foi avaliar in vitro a compatibilidade entre as resinas compostas Herculite XR, Z100, AP.H e os diferentes adesivos XR-Bond, Scotchbond MP e PUB 3, uma vez que, por uma série de motivos, é muito freqüente na prática clínica do cirurgião-dentista a utilização de resina e adesivo de marcas comerciais diferentes. Utilizou-se um dispositivo constituído de base e matriz, as quais, quando justapostas, apresentavam uma cavidade em forma de halteres que recebeu a resina composta. Inicialmente, confeccionaram-se 45 meio-espécimes de cada resina. Após uma semana armazenados em água destilada a $37^{\circ} \mathrm{C}$, os meio-espécimes foram reposicionados na matriz para confecção da segunda metade e divididos em grupos de modo a combinar cada resina com cada um dos três adesivos. Armazenaram-se os espécimes em água destilada a $37^{\circ} \mathrm{C}$ por 7 dias e procedeu-se ao teste de tração na máquina Kratos. Concluiu-se que: de forma geral, as interações entre as resinas e os adesivos testados mostraram-se compativeis; a combinação AP.H/XR-Bond apresentou a maior discrepância, pois a resistência à tração foi estatisticamente inferior à associação original proposta pelo fabricante; as demais combinações entre os adesivos e as resinas testadas apresentaram resistência à tração estatisticamente semelhantes ou superiores à associação recomendada pelo fabricante.
\end{abstract}

UNITERMOS: Adesivos; Resistência a tração; Resinas compostas.

\section{INTRODUÇÃO}

O grande desafio da Odontologia Estética e Restauradora tem sido a obtenção de um material com características estéticas e funcionais semelhantes às da estrutura dentária. Almeja-se uma adesão verdadeira deste material restaurador às da estrutura dentária, ou seja, uma adesão forte e durável mesmo nas condições adversas de umidade e variações térmicas da cavidade bucal. A corrida para a descoberta de um sistema adesivo ideal, ao esmalte e à dentina, tem ocasionado o lançamento no comércio de vários sistemas a cada $\mathrm{ano}^{5,21}$. Os sistemas adesivos recentemente introduzidos são sistemas multiuso, ou seja, segundo o fabricante, são próprios para múltiplas indicações, com a aplicação em diferentes substratos (esmalte, dentina/cemento, metais, amálgama, porcelana). Nota-se, freqüentemente, na prática clínica, que o cirurgião-dentista faz permutas entre os sistemas adesivos e as resinas compostas, ou seja, utiliza sistema adesivo multiuso e resina composta de marcas comerciais diferentes, ou mesmo emprega o sistema multiuso com uma resina composta com a qual o fabricante não recomenda ou fornece o mesmo, desconhecendo as possiveis conseqüências dessa nova combinação sobre a qualidade e durabilidade da restauração.

Percebe-se que, na maior parte das abordagens na literatura, imagina-se e analisa-se a adesão entre o material restaurador e o tecido dentário ${ }^{1,18,22,25}$. No entanto, o estudo da adesão de um material restaurador qualquer a outros materiais faz-se necessário, pois traria vantagens práticas, especialmente no contexto reparativo. Este já está alicerçado, sendo o reparo indicado para correção de contornos ou defeitos superficiais ${ }^{3,6,7,9,12}$, na técnica restauradora incremental e, atualmente, na aplicação dos sistemas adesivos multiuso com dife-

\footnotetext{
* Professores Associados do Departamento de Dentística da Faculdade de Odontologia de Bauru da USP.

** Professora Assistente da Disciplina de Dentística da Faculdade de Odontologia de Lins da UNIMEP.
} 
FRANCO, E. B.; PAZIM, M. S. L.; FRANCISCHONE, C. E. Avaliação in vitro da resistência de união de diferentes combinações entre adesivos e resinas compostas. Pesqui Odontol Bras, v. 14, n. 3, p. 225-231, jul./ set. 2000.

rentes marcas comerciais de resina composta. Alguns autores como BASSIOUNY; YING ${ }^{4}$ (1984), ROBINSON $^{19}$ (1988), BROOME ${ }^{8}$ (1985) e recentemente GONÇALVES; ARAUJO ${ }^{13}$ (1994) detiveramse nesse aspecto e pesquisaram o efeito da permuta entre adesivos dentinários e resina composta. Essa permuta pode ocorrer por vários motivos: por conveniência, uma vez que dificilmente sistema adesivo e resina composta são esgotados simultaneamente, e neste momento o profissional pode adquirir um sistema adesivo mais recente, eventualmente de marca comercial diferente para utilizar com a resina composta de que já dispunha; para melhor solucionar os problemas estéticos, especialmente no que se refere à cor, visto que muitas vezes o profissional é obrigado a recorrer a diferentes escalas de cores de variadas marcas comerciais para resolver o problema estético do paciente; involuntariamente, quando da realização de um reparo para correção de forma ou cor, em que se desconhece a marca comercial da resina composta original; para redução dos custos operacionais podendo o profissional adquirir um único sistema adesivo multiuso para ser usado com diferentes resinas compostas; para utilizar um sistema adesivo com melhor e maior resistência.

Devido à carência de estudos e dados na literatura e à importância de uma resposta esclarecedora com base científica aos cirurgiões-dentistas clinicos, planejou-se este trabalho de pesquisa com o objetivo de avaliar in vitro a resistência de união de diferentes combinações entre adesivos dentinários e resina composta.

\section{MATERIAL E MÉTODOS}

Os materiais selecionados para esta pesquisa foram as resinas compostas fotopolimerizáveis Herculite XR (Kerr), Z100 (3M) e Prisma AP.H (Dentsply) e seus respectivos adesivos, a saber: XR-Bond, Scotchbond Multi-Purpose e Prisma Universal Bond 3.

Quanto aos sistemas adesivos, somente a composição do adesivo propriamente dito foi de interesse, apresentando-se a mesma bastante diferente nos três produtos selecionados, como pode-se observar no Quadro 1.

Para confecção dos corpos-de-prova, empregou-se um dispositivo de aço inoxidável, constituído de base, matriz e contra-matriz (Figura 1, A, B e C). A matriz era formada por duas metades iguais retangulares, as quais, quando justapostas, reproduziam na parte central uma cavidade em forma de halteres, cujas dimensões estão mostradas na Figura 1. Esta cavidade que acomodou a resina composta apresentava exatamente na metade do seu comprimento uma reentrância que imprimia uma constrição no centro do corpo-de-prova, coincidente com a interface adesivo/resina composta. A base apresentava a forma de um disco com uma canaleta fresada, correspondente à metade da sua espessura, que alojava e unia as duas partes da matriz, a qual, por sua vez, recebia a contra-matriz em forma de meio-halteres no seu interior, formando o molde e mantendo-o em posição estática.

Inicialmente, com o dispositivo completo em posição, confeccionaram-se 45 meio-espécimes de cada resina composta selecionada. A resina foi inserida em dois incrementos sendo cada um polimerizado em dois pontos distintos - na cabeça e no intermediário do halteres - por $40 \mathrm{~s}$ em cada ponto, com a fonte de luz Heliomat (Vigodent S. A. Indústria e Comércio, Rio de Janeiro, Brasil). Para padronização e controle da potência do aparelho fotopolimerizador, foi utilizado um radiômetro (Demetron Research) no início de cada período de trabalho. A polimerização do primeiro incremento foi feita com a ponta do fotopolimerizador apoiada na matriz metálica e a polimerização do segundo incremento foi feita com a ponta do aparelho sobre a laminula, colocada para permitir uma superficie lisa e uniforme. A polimerização foi complementada na parte inferior do corpo-de-prova por $30 \mathrm{~s} \mathrm{em}$ cada ponto. Os meio-espécimes foram então armazenados em água destilada a $37^{\circ} \mathrm{C}$ por 7 dias. Após este período, todos os meio-espécimes receberam o seguinte tratamento na extremidade livre para aplicação do adesivo: lixamento com disco Sof-lex, aplicação de ácido fosfórico a 37\% em forma de gel, por 15 s e lavagem com jato d'água por $30 \mathrm{~s}$.

QUADRO 1 - Descrição e composição dos adesivos utilizados.

\begin{tabular}{|l|l||}
\hline \multicolumn{1}{|c|}{ Marca comercial } & \multicolumn{1}{|c|}{ Adesivo } \\
\hline XR-Bond (Kerr) & $\begin{array}{l}\text { 60\% UDMA**; 30\% TEG-DMA; } \\
10 \% \text { Fosfato DMA }\end{array}$ \\
\hline Scotchbond MP (3M) & BIS-GMA*; HEMA \\
\hline Prisma Universal & $50 \%$ UDMA**; 25\% TEG-DMA; \\
Bond 3 (Dentsply) & $4,5 \%$ PENTA; 0,5\% Glutaraldeído \\
\hline
\end{tabular}

*Dados fornecidos pelo fabricante. ${ }^{* *}$ Dados fornecidos por VAN MEERBECK et al. ${ }^{24}$. 
FRANCO, E. B.; PAZIM, M. S. L.; FRANCISCHONE, C. E. Avaliação in vitro da resistência de união de diferentes combinações entre adesivos e resinas compostas. Pesqui Odontol Bras, v. 14, n. 3, p. 225-231, jul./set. 2000.

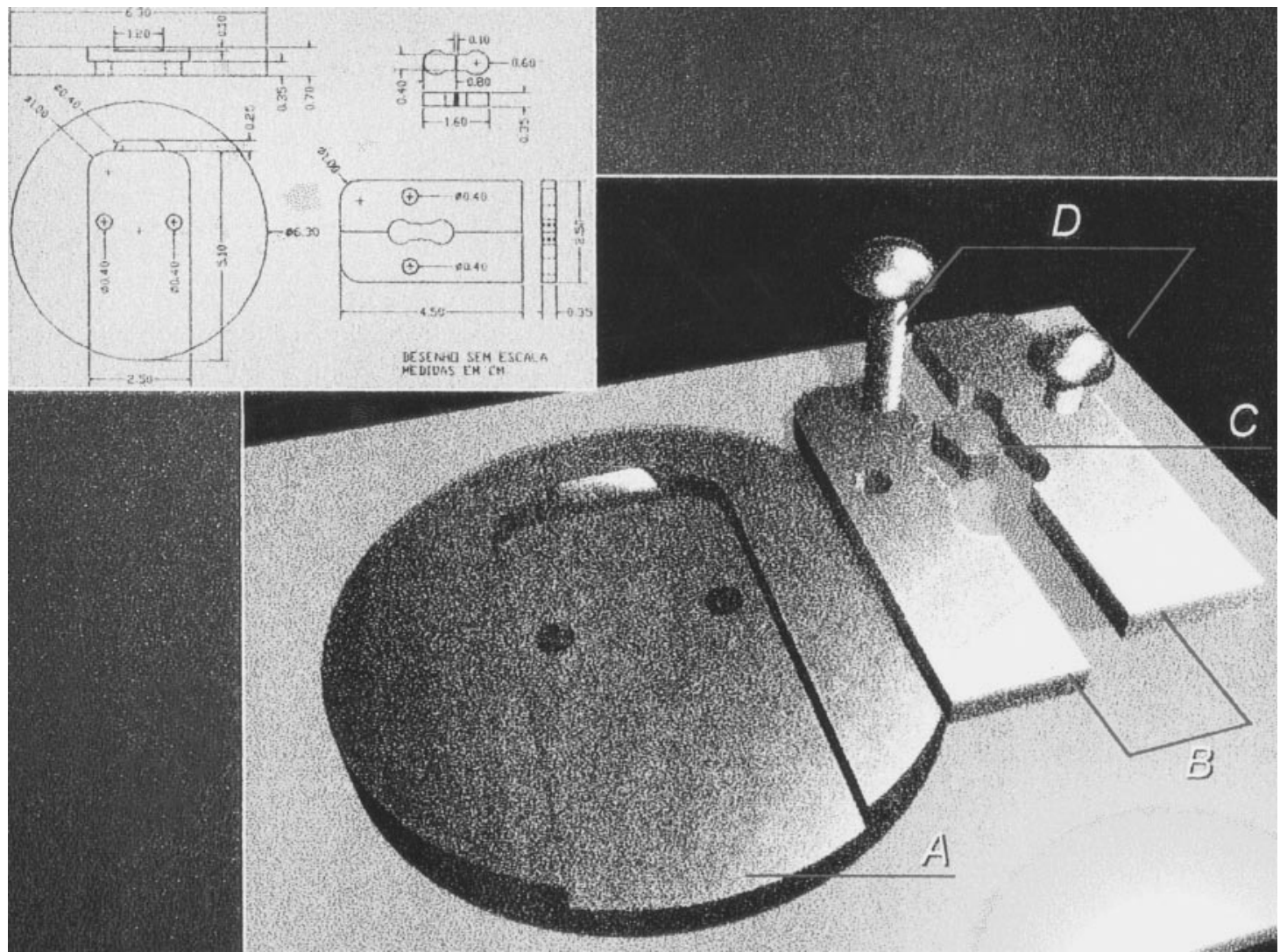

FIGURA 1 -

Dispositivo de aço inoxidável empregado para confecção de corpos-de-prova: A - Base; B - Matriz; C - Contra-matriz; D - Parafusos para fixação da matriz à base.
Após estes procedimentos, os meio-espécimes foram reposicionados na matriz, agora sem a contra-matriz, combinando a resina composta com cada um dos três adesivos, como descrito a seguir:

- Grupo A1 - Herculite XR + XR-Bond + Herculite XR.

- Grupo A2 - Herculite XR + Scotchbond MP + Herculite XR.

- Grupo A3 - Herculite XR + Prisma Universal Bond 3 (PUB 3) + Herculite XR.

- Grupo B1 - Z100 + XR-Bond + Z100.

- Grupo B2 - Z100 + Scotchbond MP + Z100.

- Grupo B3 - Z100 + PUB 3 + Z100

- Grupo C1 - Prisma AP.H + XR-Bond + Prisma AP.H.

- Grupo C2 - Prisma AP.H + Scotchbond MP + Prisma AP.H.

- Grupo C3 - Prisma AP.H + PUB 3 + Prisma AP.H.

Aplicou-se uma fina camada do adesivo designado para cada grupo, depois um leve jato de ar e em seguida polimerizou-se por 20 segundos. A segunda metade do espécime foi feita, então, inserindo-se a resina composta em duas camadas e polimerizando-se cada uma nos dois pontos por 40 segundos. Os corpos-de-prova foram então removidos do molde e a polimerização complementada da mesma forma anteriormente descrita para o primeiro meio-espécime. Dessa forma, os corposde-prova estavam concluídos.

Todos os corpos-de-prova foram armazenados em água destilada a $37^{\circ} \mathrm{C}$ por 7 dias, e então submetidos ao teste de tração, na máquina de ensaios universal (Kratos), utilizando-se a célula de carga $\mathrm{n}^{\circ} 2$, escala 1 à velocidade de $0,5 \mathrm{~mm} / \mathrm{min}$.

A adaptação dos corpos-de-prova na máquina de ensaio foi feita por meio de dispositivo de forma retangular, confeccionado em aço inoxidável com uma cavidade semicircular retentiva que servia para encaixar a extremidade do corpo-de-prova e permitir os ensaios de tração (Figura 2).

Entre a máquina de ensaio e esse dispositivo foi utilizada uma junta de aço inoxidável e um girador de latão, que simulavam uma junta universal e evitavam o efeito de torção nas amostras, o que poderia interferir nos resultados.

\section{RESULTADOS E DISCUSSÃO}

Após o teste de tração em cada corpo-de-prova e a observação macroscópica do local da fratura, ve- 
FRANCO, E. B.; PAZIM, M. S. L.; FRANCISCHONE, C. E. Avaliação in vitro da resistência de união de diferentes combinações entre adesivos e resinas compostas. Pesqui Odontol Bras, v. 14, n. 3, p. 225-231, jul./ set. 2000.

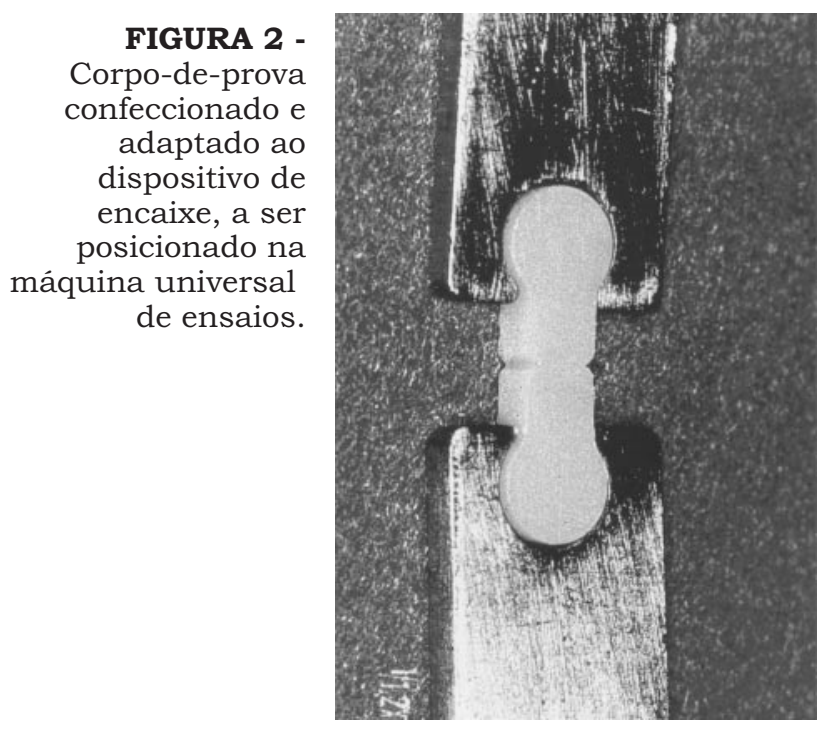

rificou-se que esta foi sempre adesiva, ou seja, na interface sistema adesivo/resina composta, ocorrendo na área correspondente à ligeira constrição da parte central da matriz. Os resultados foram submetidos à análise estatística, sendo a análise de variância a um critério o modelo adotado. As médias aritméticas e desvios-padrão de cada grupo estão expressos na Tabela 1.

Numa visão geral, das médias de resistência à tração dos 9 grupos analisados, verifica-se que os valores de resistência à tração foram maiores para a resina composta Prisma AP.H, seguida pela Z100 e Herculite, semelhantemente ao que ocorreu no estudo de PAZIM ${ }^{15}$ (1995). O fator composição, ou seja, proporção entre quantidade de partículas inorgânicas e matriz orgânica pode explicar este resultado, uma vez que a resistência de união é proporcional à "molhabilidade" (capacidade do adesivo umedecer a superficie do aderente). Um substrato com maior conteúdo orgânico permitiria uma maior "molhabilidade" e, conseqüentemente, maiores valores de resistência de união. A resina Prisma AP.H apresenta uma menor porcentagem em volume de partículas inorgânicas, o que implica em maior contingente resinoso, ou seja, matriz orgânica. O tamanho de partículas poderia também estabelecer uma correlação para aumentar ou diminuir a união entre superfícies, uma vez que partículas maiores tendem a mostrar maiores irregularidades superficiais. Isto favoreceria maior ligação mecânica, além de apresentarem maior contingente resinoso entre as partículas, o que permitiria maior interação química com o agente adesivo. Nesta explicação, considerou-se a capa-
TABELA 1 - Valores médios em kgf (MPa) da resistência à tração de corpos-de-prova e respectivos desvios-padrão, em função da interação das resinas compostas e agentes adesivos.

\begin{tabular}{c|c|c|c|c|c|c}
\hline \hline \multirow{2}{*}{ Adesivos } & \multicolumn{5}{|c}{ Resinas compostas } \\
\cline { 2 - 7 } & $\begin{array}{c}\text { Herculite XR } \\
\text { (A) }\end{array}$ & \multicolumn{2}{c}{$\begin{array}{c}\text { Z100 } \\
\text { (B) }\end{array}$} & \multicolumn{2}{c}{$\begin{array}{c}\text { Prisma AP.H } \\
\text { (C) }\end{array}$} \\
\hline & $\mathrm{X}$ & $\mathrm{DP}$ & $\mathrm{X}$ & $\mathrm{DP}$ & $\mathrm{X}$ & $\mathrm{DP}$ \\
\hline $\begin{array}{c}\text { XR-Bond } \\
(1)\end{array}$ & $\begin{array}{c}17,10 \\
(13,97)\end{array}$ & 1,68 & $\begin{array}{c}22,12 \\
(18,07)\end{array}$ & 3,34 & $\begin{array}{c}18,77 \\
(15,22)\end{array}$ & 3,45 \\
\hline $\begin{array}{c}\text { Scotchbond } \\
\text { MP } \\
(2)\end{array}$ & $\begin{array}{c}18,86 \\
(15,41)\end{array}$ & 2,23 & $\begin{array}{c}19,40 \\
(15,84)\end{array}$ & 2,96 & $\begin{array}{c}30,20 \\
(24,67)\end{array}$ & 3,90 \\
\hline $\begin{array}{c}\text { PUB 3 } \\
(3)\end{array}$ & $\begin{array}{c}15,62 \\
(12,76)\end{array}$ & 2,69 & $\begin{array}{c}21,24 \\
(17,35)\end{array}$ & 2,46 & $\begin{array}{c}27,25 \\
(22,26)\end{array}$ & 3,87 \\
\hline \hline
\end{tabular}

TABELA 2 - Comparações múltiplas entre as médias de resistência de união, por tração, com a resina composta Herculite XR e os diferentes adesivos, pelo teste de Tukey-Kramer.

\begin{tabular}{c|c|c|c}
\hline \hline Grupos & $\mathrm{n}$ & Média & Grupos homogêneos \\
\hline A (3) & 15 & 15,620000 & ns \\
\hline A (1) & 15 & 17,100000 & ns \\
\hline A (2) & 15 & 18,860000 & \\
\hline \hline
\end{tabular}

$\mathrm{n}=$ tamanho da amostra; $\mathrm{p}<0,05$;

ns = não significante;

$\mathrm{Vc}=2,33$.

cidade do adesivo molhar a superficie de resina composta (meio-espécime). Por outro lado, a capacidade da resina composta estabelecer ligações químicas com a superficie do meio-espécime coberta com adesivo também pode ter influenciado nos resultados, uma vez que uma resina com menor viscosidade conduz a um melhor molhamento, melhor contato para ligação e maiores valores de resistência de união ${ }^{9,23}$. Como aconteceu na presente pesquisa, a resina Prisma AP.H apresentou a menor viscosidade, seguida pela Z100 e a Herculite XR com a maior viscosidade.

A análise de variância a um critério, aplicada separadamente para cada resina composta, mostrou diferenças estatisticamente significantes entre os grupos, para as três diferentes combinações entre resina composta e adesivos. O teste de Tukey-Kramer foi então aplicado para todas as resinas compostas, para detectar as interações responsáveis pela diferença estatística (Tabelas 2, 3 e 4). 
FRANCO, E. B.; PAZIM, M. S. L.; FRANCISCHONE, C. E. Avaliação in vitro da resistência de união de diferentes combinações entre adesivos e resinas compostas. Pesqui Odontol Bras, v. 14, n. 3, p. 225-231, jul./set. 2000.

Com relação à resina composta Herculite XR (Tabela 2), obteve-se diferença estatisticamente significante entre os grupos A2 e A3, sendo os valores de A2 maiores que os de A3. Não houve diferença estatisticamente significante entre A1 e A2 e entre A1 e A3. Estas comparações sugerem que, preferencialmente, a resina composta Herculite XR poderia ser utilizada com os adesivos Scotchbond Multi-Purpose e com o próprio adesivo $\mathrm{XR}$-Bond, uma vez que estas combinações forneceram valores estatisticamente semelhantes.

Para a resina composta $Z 100$ (Tabela 3), houve diferenças significantes entre os grupo B1 e B2, sendo os valores de B1 superiores aos de B2. Há que se ressaltar que esta diferença ficou bem próxima ao valor crítico. As demais interações não mostraram diferenças estatisticamente significantes. Estas análises sugerem que a resina Z100 poderia ser utilizada com os três adesivos testados sem qualquer prejuízo em relação à resistência à tração. Embora estes resultados concordem com outros estudos $^{12,19,23}$, PRATI; MONTANARI ${ }^{17}$ (1988); ROBINSON $^{19}$ (1988) não recomendam a permuta entre sistemas adesivos.

Quanto à Prisma AP.H (Tabela 4), a diferença estatística ocorreu entre duas comparações: os valores de $\mathrm{C} 2$ foram superiores aos de $\mathrm{C} 1$ e os valores de C3 superiores aos de C1. Sugere-se, deste modo, que a resina composta Prisma AP.H deveria ser associada, preferencialmente, aos adesivos Scotchbond MP e PUB 3. Parece haver uma menor compatibilidade entre a resina composta Prisma AP.H e o adesivo XR-Bond. Estes resultados estão de acordo com outros estudos de ALBERS² (1990); GONÇALVES $^{13}$ (1994). Estas comparações estatísticas não podem ser analisadas isoladamente para contra-indicar a combinação Prisma AP.H/XRBond. Observa-se que a média de resistência de união da associação Prisma AP.H/XR-Bond foi nu-

TABELA 3 - Comparações múltiplas entre as médias de resistência de união, por tração, com a resina composta Z100 e os diferentes adesivos, pelo teste de Tukey-Kramer.

\begin{tabular}{c|c|c|c}
\hline \hline Grupos & $\mathrm{n}$ & Média & Grupos homogêneos \\
\hline $\mathrm{B}(2)$ & 15 & 19,400000 & $\mathrm{~ns}$ \\
\hline $\mathrm{B}(3)$ & 15 & 21,240000 & $\mathrm{~ns}$ \\
\hline $\mathrm{B}(1)$ & 15 & 22,120000 & \\
\hline \hline
\end{tabular}

$\mathrm{n}=$ tamanho da amostra; $\mathrm{p}<0,05$;

ns $=$ não significante;

$\mathrm{Vc}=2,62$. mericamente comparável ou maior à dos demais grupos, A e B, testados no presente trabalho. As variações na composição química e pureza dos sistemas comerciais de resinas compostas e adesivos testados em trabalhos laboratoriais ou clínicos são comumente apontados como justificativas ou razões prováveis para os resultados obtidos ${ }^{10,11,16,20,22}$. Selecionaram-se, no trabalho em questão, resinas e adesivos com composições diferentes justamente para pesquisar a compatibilidade entre as diferentes formulações. A dificuldade, entretanto, é o desconhecimento da composição real dos diferentes produtos comerciais, o que acaba gerando algumas dúvidas quanto às suas propriedades e/ou comportamento ${ }^{20}$.

Destacam-se alguns prováveis fatores que influenciaram nas ligações adesivas, como índice de conversão, quantidade de ligações duplas remanescentes e a quantidade de monômero residual. O indice de conversão é um elemento considerável na medida em que reflete a eficácia da polimerização. Para as resinas compostas fotopolimerizáveis, o mesmo depende da eficácia da fonte de luz, do sistema iniciador, da estrutura dos monômeros e da capacidade dos metacrilatos se difundirem através da matriz polimérica e reagirem com radicais terminais da cadeia, tipo e quantidade das partículas de carga, uma vez que a dispersão da luz nas resinas compostas ocorre devido às partículas de carga e distância da fonte de luz, a qual não pode exceder a $2 \mathrm{~mm}^{20,25}$. Para o presente trabalho, utilizou-se sempre o mesmo fotopolimerizador e observou-se a distância mais próxima possível para polimerização da resina composta.

Alguns estudos ${ }^{20,25}$ concordam que para resina composta contendo os monômeros BIS-GMA e TEG-DMA pode-se fazer a seguinte correlação: quanto maior a quantidade de BIS-GMA e menor a quantidade de TEG-DMA, menor a conversão e menor a difusão de radicais para ligação cruzada,

TABELA 4 - Comparações múltiplas entre as médias de resistência de união, por tração, com a resina composta Prisma AP.H e os diferentes adesivos, pelo teste de Tukey-Kramer.

\begin{tabular}{c|c|c|c}
\hline \hline Grupos & $\mathrm{n}$ & Média & Grupos homogêneos \\
\hline $\mathrm{C}(1)$ & 15 & 18,773333 & \\
\hline $\mathrm{C}(3)$ & 15 & 27,253333 & $\mathrm{~ns}$ \\
\hline $\mathrm{C}(2)$ & 15 & 30,206667 & $\mathrm{~ns}$ \\
\hline \hline
\end{tabular}

$\mathrm{n}=$ tamanho da amostra; $\mathrm{p}<0,05$;

ns = não significante;

$\mathrm{Vc}=3,33$. 
FRANCO, E. B.; PAZIM, M. S. L.; FRANCISCHONE, C. E. Avaliação in vitro da resistência de união de diferentes combinações entre adesivos e resinas compostas. Pesqui Odontol Bras, v. 14, n. 3, p. 225-231, jul./ set. 2000.

com conseqüente redução da taxa de polimerização e grande quantidade de ligações duplas remanescentes. No Quadro 1, pode-se observar variações na composição dos diferentes adesivos que em maior ou menor grau podem influenciar na interação adesiva.

\section{CONCLUSÕES}

Após análise estatística e discussão dos resultados obtidos, pode-se concluir que:

1. de forma geral, as interações entre as resinas compostas Herculite XR, Z100 e Prisma AP.H e os adesivos XR-Bond, Scotchbond MP e PUB 3 mostraram-se compativeis;

2. a combinação Prisma AP.H/XR-Bond apresentou a maior discrepância, visto que mostrou resistência à tração estatisticamente inferior à associação original, proposta pelo fabricante;

3. as demais combinações entre os adesivos XR-Bond, Scotchbond MP e PUB 3 e cada uma das resinas compostas Herculite XR, Z100 e Prisma AP.H apresentaram resistência à tração estatisticamente semelhantes ou superiores à associação recomendada pelo fabricante.

FRANCO, E. B.; PAZIM, M. S. L.; FRANCISCHONE, C. E. In vitro evaluation of the bond strength of different combinations of adhesives and composite resins. Pesqui Odontol Bras, v. 14, n. 3, p. 225-231, jul./set. 2000.

The aim of this work was to evaluate the in vitro compatibility between some composite resins (Herculite XR, Z100 and AP.H) and different adhesives (XR-Bond, Scotchbond MP and PUB3), since for various reasons, the utilization of composite resins and adhesives from different commercial brands is very frequent in the dental practice. We used a device constituted of a base and a matrix which, when juxtaposed, showed a cavity in dumbbell shape which received the composite resin. Firstly, 45 half-specimens of each composite resin were made and stored in distilled water at $37^{\circ} \mathrm{C}$ for one week. Then, they were replaced in the matrix for the building of the second half and divided into groups to combine each composite resin with each one of the three adhesives. The specimens were stored in distilled water at $37^{\circ} \mathrm{C}$ and after the period of 7 days they were submitted to the tensile strength test in a Kratos machine. It was concluded that: generally, the composite resins and the adhesives showed to be compatible; the combination AP.H/XR-Bond showed the highest discrepancy, since the tensile strength was statistically smaller than that of the combination proposed by the manufacturer; the other combinations of the adhesives and the composite resins showed tensile strengths statistically similar to or greater than that of the combination recommended by the manufacturer.

UNITERMS: Adhesives; Tensile strength; Composite resins.

\section{REFERÊNCIAS BIBLIOGRÁFICAS}

1. ABDALLA, A. I.; DAVIDSON, C. L. Shear bond strength and microleakage of new dentin bonding systems. Am J Dent, v. 6, n. 6, p. 295-298, Dec. 1993.

2. ALBERS, H. F. Pertinent information on cosmetic, adhesive and restorative dentistry. Adept report, v. 1, n. 4, p. 33-44, Fall 1990.

3. ARIETA, M. L. Avaliação da resistência de união de reparos realizados com resinas compostas. Bauru, 1989. 93 p. Dissertação (Mestrado) - Faculdade de Odontologia de Bauru, Universidade de São Paulo.

4. BASSIOUNY, M.; YING, L. Adhesive compatibility of restorative resins with dentin bonding agents. J Dent Res, v. 63, special issue, p. 232, 1984. [Abstract n. 553]

5. BAYNE, S. C. Update on dental composites restorations. J Am Dent Assoc, v. 125, n. 6, p. 687-701, June 1994.

6. BOYER, D. B. Build-up and repair of light-cured composites: bond strength. J Dent Res, v. 63, n. 10, p. 1241-1244, Oct. 1984.

7. BOYER, D. B. The strength of multilayer and repaired composite resin. J Prosthet Dent, v. 39, n. 1, p. 63-67, Jan. 1988.

8. BROOME, J. C. Shear bond strengths of composite resin with three dentin adhesives. J Dent Res, v. 64, p. 244, 1985. /Abstract n. 622/

9. CAUSTON, B. E. Repair of abraded composite fillings. Br Dent J, v. 139, n. 7, p. 286-288, Oct. 1975.

10. CHIBA, K. The addition of an adhesive composite resin to the same material: bond strength and clinical techniques. J Prosthet Dent, v. 61, n. 6, p. 669-675, June 1989.

11. CHIN, Y. H. The bond strength of incrementally placed composite resins. Aust Dent J, v. 32, n. 4, p. 247-251, 1987.

12. ELI, I. Sequentially light-cured composites: strength of bond between layers. J Prosthet Dent, v. 56, p. 158-161, 1986.

13. GONÇALVES, S. E. P.; ARAUJO, M. A. M. Avaliação mecânica e microscópica de sistemas adesivos multiuso. Anais da Sociedade Brasileira de Pesquisas Odontológicas, v. 10, p. 124, set. 1994. /Resumo no 243/

14. NAKABAYASHI, N. Hybrid layer as a dentin-bonding mechanism. J Esthet Dent, v. 3, n. 4, p. 133-138, July/Aug. 1991.

15. PAZIM, M. S. L. Avaliação da resistência de união de resinas compostas em função da técnica de inserção. Anais da Sociedade Brasileira de Pesquisas Odontológicas, v. 11 , p. 53, set. 1995. [Resumo n. 106] 
FRANCO, E. B.; PAZIM, M. S. L.; FRANCISCHONE, C. E. Avaliação in vitro da resistência de união de diferentes combinações entre adesivos e resinas compostas. Pesqui Odontol Bras, v. 14, n. 3, p. 225-231, jul./set. 2000.

16. PEUTZFELDT, A. Correlation between recordings obtained with a light-intensity tester and degree of conversion of a light-curing resin. Scand J Dent Res, v. 102, p. 73-75, 1994.

17. PRATI, C.; MONTANARI, G. The effect of microleakage of interchanging denture adhesives in two composite resin systems in vitro. Br Dent J, v. 164, p. 273, May 1988.

18. RETIEF, D. H. Adhesion to dentin. J Esthet Dent, v. 3, n. 3, p. 106-13, May/June 1991.

19. ROBINSON, P. B. The effect on microleakage of interchanging dentine adhesives in two composite resin systems in vitro. Br Dent J, v. 164, n. 3, p. 77-79, Feb. 1988.

20. RUYTER, I. E. Monomer systems and polimerization. In: VANHERLE, G.; SMITH, D. C. Posterior composite resin dental restorative materials. St. Paul, Dental Products Division 3M Company, 1985. p. 109-136.
21. SILVA e SOUZA Jr., M. H. Adesivos dentinários: evolução, estágio atual e considerações clínicas para sua utilização. Maxi-odonto: Dentística, v. 1, n. 1, p. 1-18, jan./fev. 1995.

22. TRIOLO Jr., P. T. Shear bond strengths of composite to dentin using six dental adhesive systems. Oper Dent, v. 20, p. 46-50, 1995.

23. VANKERCKHOVEN, H. Unreacted methacrylate groups on the surfaces of composite resins. J Dent Res, v. 61, n. 6, p. 791-796, June 1982 .

24. VAN MEERBEEK, B. Factors affecting adhesion to mineralized tissues. Oper Dent, p. 111-124, 1992. Supplement 5.

25. VENHOVEN, B. A. M. Polymerization contraction and conversion of light-curing Bis-GMA based methacrylate resins. Biomaterials, v. 14, n. 11, p. 871-875, 1993.

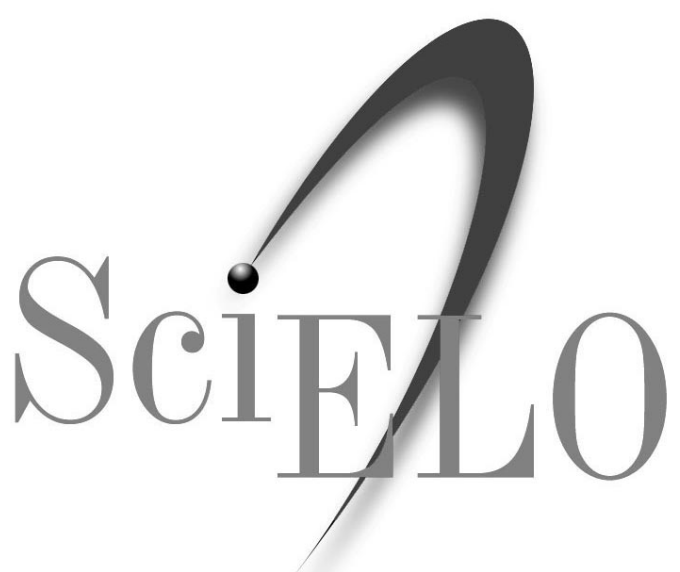

\section{Scientific Electronic Library Online http://www.scielo.br Parceria: FAPESP/BIREME}

SciELO -Scientific Electronic Library Online é uma coleção eletrônica de revistas científicas disponíveis na Internet. A coleção traz os textos completos de artigos científicos, bases de dados e relatórios de uso e de impacto da literatura científica publicada no site.

Em operação desde 1997, atualmente estão disponíveis 29 revistas brasileiras nas áreas de ciências biológicas e da saúde, ciências sociais e humanas, ciências agrárias, física etc. Novas publicações são incluídas regularmente em várias especialidades.

Entre as revistas de nossa coleção, está a Pesquisa Odontológica Brasileira.

$$
\begin{aligned}
& \text { Consulte a Pesquisa Odontológica Brasileira (Revista de Odontologia da } \\
& \text { USP) na SciELO - http:/ / www.scielo.br / rousp }
\end{aligned}
$$

